\title{
Performance prediction of a centrifugal pump working in direct and reverse mode using Computational Fluid Dynamics
}

\author{
R. Barrio ${ }^{1}$, J. Fernández $^{2}$, J. Parrondo ${ }^{1}$ and E. Blanco ${ }^{1}$ \\ ${ }^{1}$ Departamento de Energía \\ E.P.S.I.G., Universidad de Oviedo \\ Campus de Viesques s/n, 33271 Gijón (Spain) \\ Phone: +34 9851822 84, Fax: +34 9851820 98, E-mail: barrioraul@uniovi.es \\ 2 Departamento de IMEM \\ Escuela de Ingenierías Industriales, Universidad de Extremadura \\ Avda. de Elvas s/n, 06071 Badajoz (Spain) \\ Phone: +34 9242896 11, Fax: +34 9242896 01, E-mail: ffrancos@unex.es
}

\begin{abstract}
The operation of centrifugal pumps as centripetal turbines can be useful to produce cheap energy in rural zones or developing nations. However, the optimum coupling between the flow and the geometry of the machine cannot be kept in reverse working conditions, and hence the flow instabilities are expected to be much higher. This paper presents a numerical investigation on the unsteady flow in a commercial centrifugal pump operating in direct (pump) and reverse (turbine) mode. Numerical simulations were conducted for several flow rates to investigate the properties of the global flow within the machine. The numerical model was additionally used to estimate the radial load on the impeller, as a function of flow rate, for both modes of operation.
\end{abstract}

\section{Key words}

Centrifugal pump; CFD simulations; reverse running; unsteady flow; radial load.

\section{Introduction}

Centrifugal pumps can extract energy from a flow of water to supply power to a generator [1], thus operating as centripetal turbines. This mode of operation can be advantageous to produce cheap energy in rural zones or developing nations.

The geometry of the pumps is usually designed to get an optimum coupling between the impeller and the volute at the so-called nominal flow rate [2]. In this situation, the flow matches suitably the geometry of the machine, and thus the flow instabilities are reduced to a minimum. In contrast, these optimum coupling conditions cannot be kept when the pump operates in reverse mode at the same flow rate.

When operating as a centripetal turbine, it is observed that the point of maximum efficiency shifts towards a higher flow rate than that corresponding to direct (pump) mode. Additionally, and due to the shape of the efficiency characteristic (which is quite flat for high flow rates), it is recommended that the operating flow rate in reverse mode will be greater than the design flow rate of the pump [3]. In consequence, the flow instabilities and the radial unbalance generated when working as a centripetal turbine are expected to be much higher.

The Computational Fluid Dynamics (or CFD) programs are gaining in importance for the design and analysis of fluid turbomachinery. The numerical analysis usually implies full 3D simulations to account for turbulent effects, secondary flows, and unsteady phenomena. For the particular case of centrifugal pumps, it is known that the unsteady nature of the flow generates an unsteady interaction with the volute that affects the performance of the machine [4]. This interaction brings about pressure pulsations that generate an unsteady load on the mechanical parts [4-6], thus constituting one of the major sources of vibration and hydraulic noise [7].

This paper presents a numerical investigation on the performance of a commercial centrifugal pump operating in direct and reverse mode. The numerical calculations were carried out with the code FLUENT. The dependence of the numerical predictions in regard to grid and time step size was checked first. The model was subsequently validated with experimental data of global performance characteristics and, once validated, it was used to investigate the global flow and also to estimate the radial thrust on the impeller as a function of flow rate.

\section{Numerical model}

The model was developed for a conventional centrifugal pump with volute casing. The impeller has an eye diameter of $52 \mathrm{~mm}$, outer diameter of $200 \mathrm{~mm}$ and seven blades of logarithmic profile. The width at the outlet of 
the impeller is $0.0169 \mathrm{~m}$ and the blade outlet angle is 29 deg. The minimum radial gap between the impeller and volute tongue is $17 \%$ of outer radius. The nominal performance values (pump mode) are $\phi=0.011$ for flow rate and $\psi=0.16$ for head. The non-dimensional specific speed is $n_{\mathrm{s}}=0.42$.

Fig. 1 presents a picture of the numerical model showing the surface mesh. The walls of the casing and also the inlet duct are removed in order to show the geometry of the impeller. The mesh is unstructured and constituted by tetrahedral cells in the impeller and volute and by prismatic cells in the inlet and outlet duct. The total number of cells was established in about $8 \cdot 10^{5}$ after a grid dependence analysis. The maximum cell skew resulted to be 0.85 .

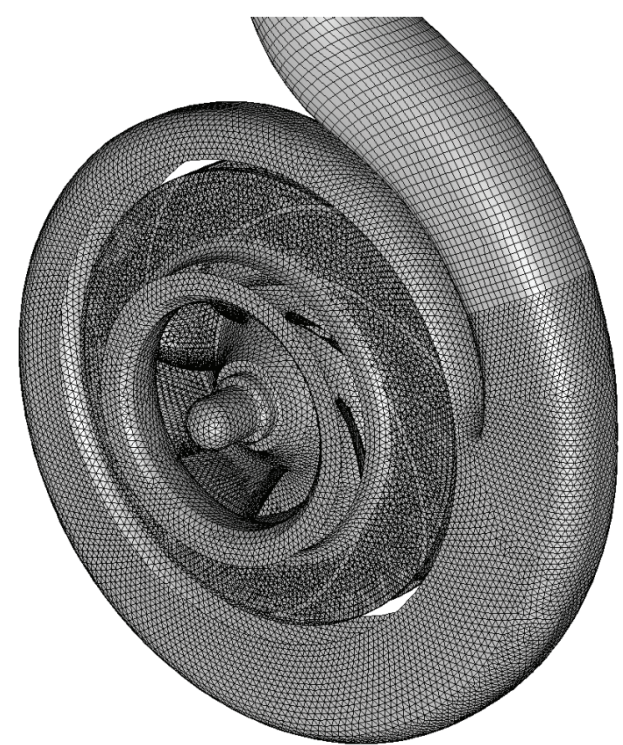

Fig. 1. Global view of the pump surface mesh.

The numerical calculations were carried out with the commercial code FLUENT by the finite volume method. This code was used to solve the full unsteady Reynoldsaveraged Navier-Stokes equations together with the standard k-epsilon model plus standard wall functions to simulate turbulent effects. The boundary conditions imposed in pump mode were a constant total pressure at the inlet and a variable static pressure at the outlet. In turbine mode, a constant total pressure at the inlet and a constant static pressure at the outlet were chosen. These boundary conditions avoid the definition of a fixed velocity profile at the inlet and outlet sections, and thus they allow for some flow rate pulsation, which is a feature of real pumps. Second order discretizations were used for the convection and diffusion terms. The SIMPLE algorithm established the pressure-velocity coupling. The relative motion between the impeller and the stationary zones of the domain was imposed by defining a grid interface at each fluid region. A sliding mesh technique, which is supported in FLUENT, uses an interpolation routine to exchange data of the cells at both sides.

The simulations were carried out first using a frozenrotor interface (i.e. for a relative angular position of the impeller). Once steady convergence was achieved, the resulting velocity and pressure fields were used as initial conditions for the unsteady calculations. The time step size used for the calculations was established in $1.65 \times 10^{-4}$ seconds after a time dependence analysis, with resulted in a total of 224 time steps per impeller revolution (i.e. a change of $1.6 \mathrm{deg}$ in the impeller position between two consecutive time steps). The number of iterations in each time step was adjusted to reduce the scaled residuals below $10^{-5}$. At least 5 impeller revolutions were required to achieve unsteady convergence, in the sense that the flow variables became periodic when considering one full revolution.

\section{Experimental contrast}

The calculations were performed for five flow rates in each mode of operation. The predictions from the numerical model were subsequently validated with experimental data collected at laboratory. The laboratory tests were carried out using a hydraulic rig that permitted the pumping of the water from and to a reservoir of big dimensions. The magnitude of flow rate was obtained with an electromagnetic flow-meter with an estimated precision of $\pm 0.8 \%$. Two piezorresistive pressure transducers installed at the inlet and outlet duct were used to calculate the head with an estimated precision of $\pm 1 \%$. The tests were carried out at a constant speed of $1750 \pm 1$ $\mathrm{rpm}$. An auxiliary pump, connected in series with the test pump, was used for the reverse running tests to supply the flow rate and head required. This pump was driven by a motor controlled by a frequency converter.

The experimental flow-head characteristic in direct (pump) and reverse (turbine) mode of operation and the predictions obtained from the numerical model are shown in Fig. 2. As observed, the general trend of the curves is suitably predicted by the model, showing a negative slope with flow rate for direct mode and a positive slope for reverse mode. It is also seen that the numerical predictions are in very good agreement with the experimental values, showing maximum (relative) errors less than $4 \%$.

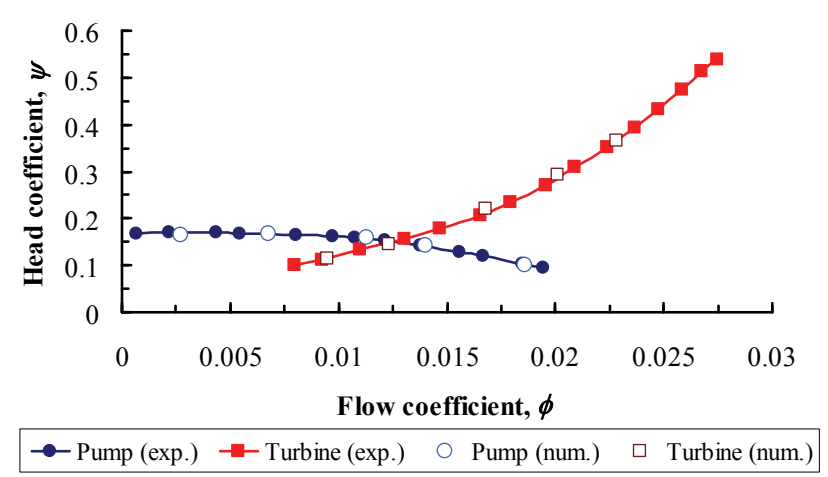

Fig. 2. Flow-head characteristic in direct and reverse mode.

\section{Analysis of global flow}

Once validated, the numerical model was used to investigate the global flow in the machine for both modes of operation. Fig. 3 presents an instantaneous contour 
map of the static pressure at the near-tongue region, obtained at impeller mid-span, for the $60 \%, 100 \%$, and $130 \%$ of pump rated flow. The relative velocity vectors are also included in the figure. It is observed that the static pressure increases continuously along the impeller passageways from the inner to the outer region as energy is transmitted due to the impulse of the blades. The pressure reaches higher values at the lowest flow rate while decreasing with increasing flow rates, as expected for a centrifugal pump (see Fig. 2). A low pressure region is noted near the tongue for the $60 \%$ flow rate. This region is located between the tongue and the impeller, as can be seen in the top image. In contrast, the highest flow rate ( $130 \%$ of rated) shows a low pressure region close to the exit duct and, additionally, a high pressure region between tongue and impeller. The flow progresses smoothly through this volute region at nominal flow rate.

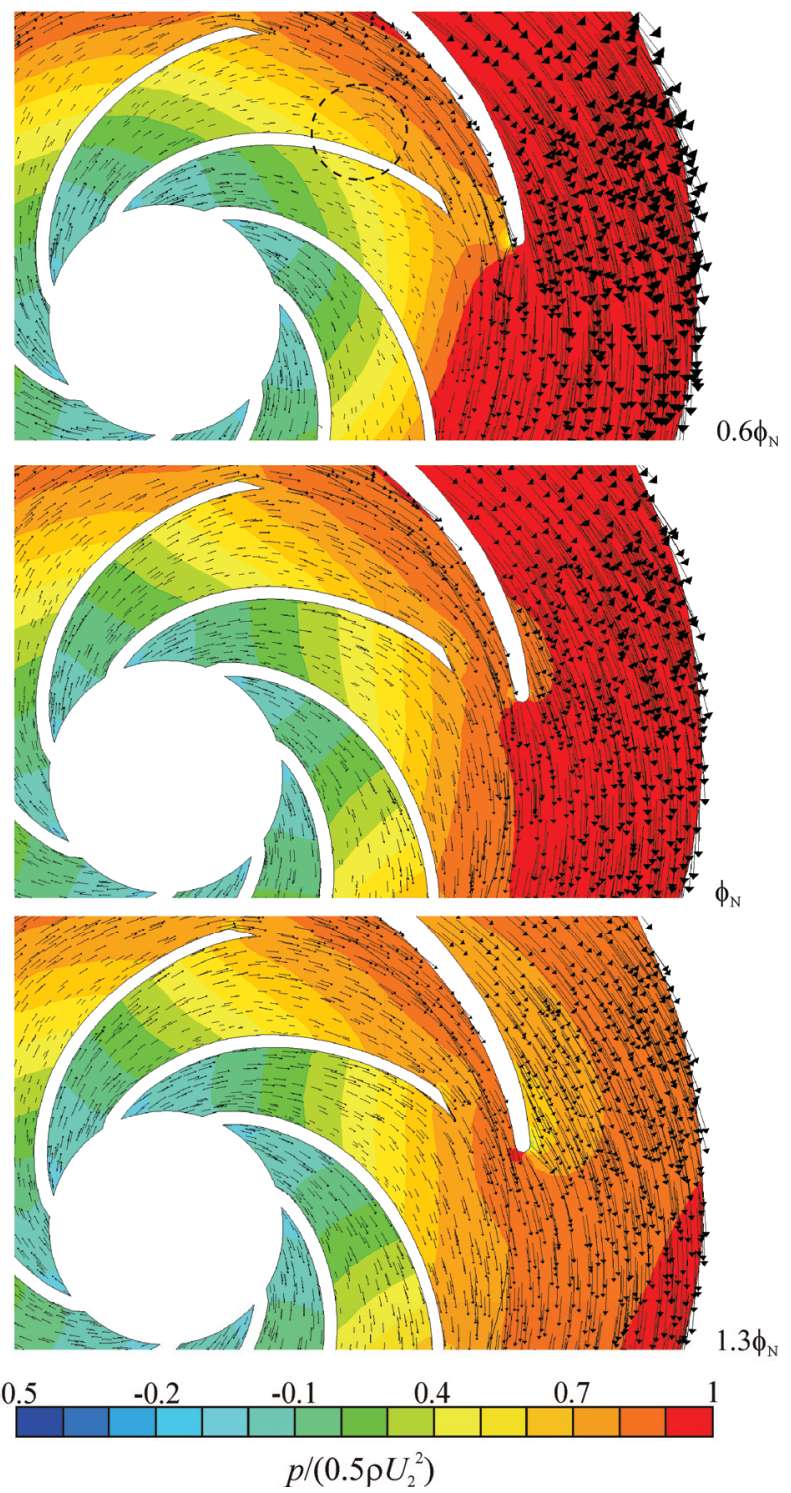

Fig. 3. Instantaneous map of static pressure at the near-tongue region and relative velocity vectors for three test flow rates (direct mode of operation).

The relative velocity vectors show that there is a good guidance of the flow along the passageways for medium and high flow rates, showing streamlines adhered to the blade walls. At the lowest flow rate, in contrast, it is observed that there is a region located near the blade pressure side (dashed circle) where the flow reverses direction, thus constituting a re-circulating bulk of fluid. This recirculation provokes a pressure drop that changes pressure direction in the last portion of the blade (i.e. there is a higher pressure on the suction side than on the pressure side). In consequence, this blade does not transmit energy to the fluid in its last portion when passing near the tongue.
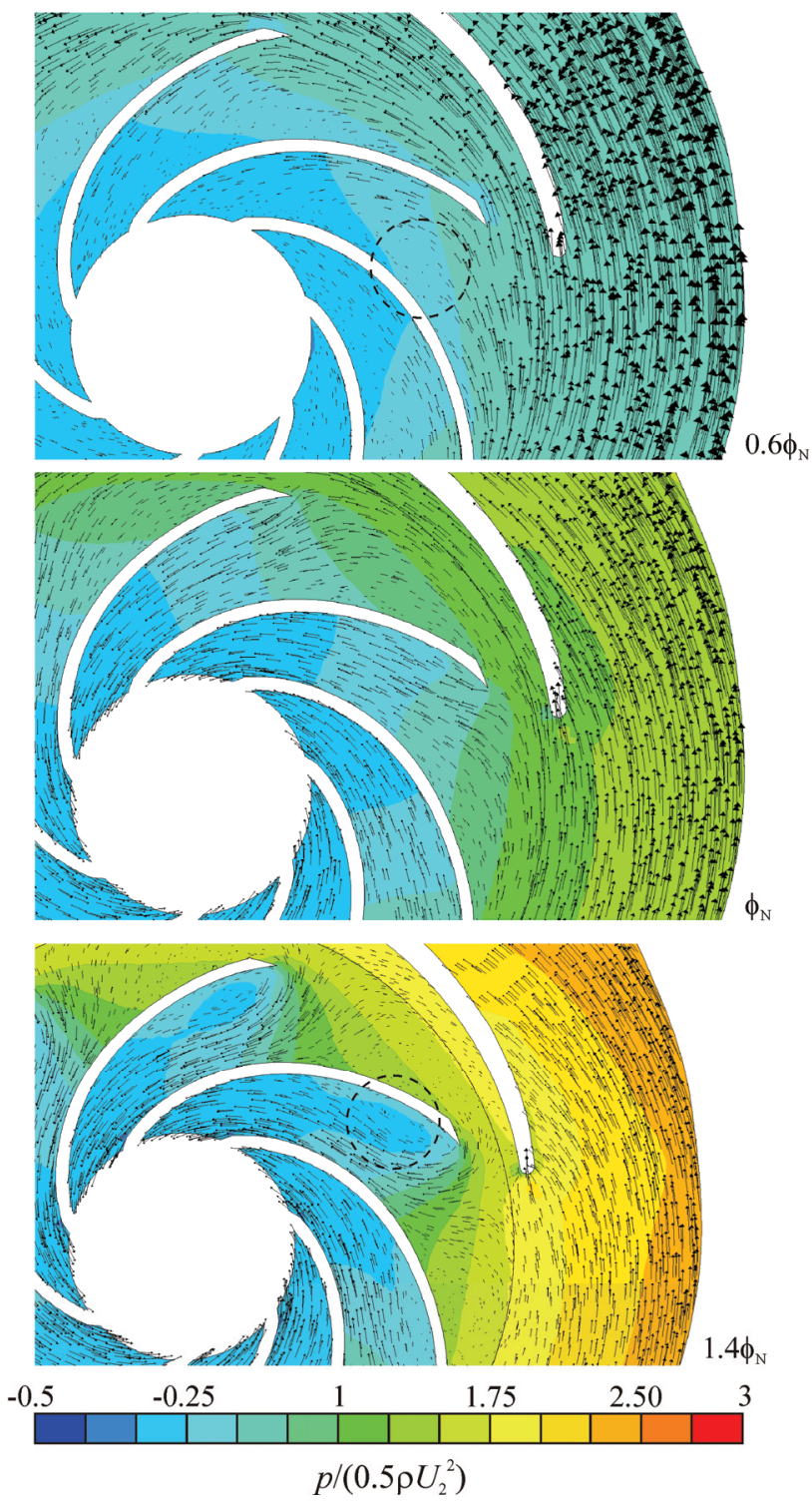

Fig. 4. Instantaneous map of static pressure at the near-tongue region and relative velocity vectors for three test flow rates (reverse mode of operation).

Fig. 4 presents equivalent results for similar flow rates of those shown in Fig. 3. In the present case, however, the pump operates in reverse mode (centripetal turbine). As noted, the pressure diminishes from the outer to the inner section of the impeller due to the continuous transmission of energy from fluid to blades. The net head across the impeller increases with increasing flow rates, as expected for a centripetal turbine (see characteristics of Fig. 2). While the pressure seems quite uniform at the tongue region for the $60 \%$ flow rate, it can be seen that there is a 
low pressure region between the impeller and the tongue for the two higher flow rates.

The analysis of the relative velocity vectors shows that the guidance of the flow across the passageways is not as adequate as it was previously in pump mode. Thus, it can be noted that the predicted nominal flow rate presents the best guidance among the results shown in Fig. 4 but, nonetheless, a small re-circulating region can be observed near the trailing edge of the blades (pressure side). This region increases in magnitude and it extends further down the inner section of the impeller at $60 \%$ flow rate, thus causing the incoming fluid to move closer to the suction side of the blades, as observed in the top image of Fig. 4. At $140 \%$ flow rate, the increasing angle of the incoming fluid causes the re-circulating region to move closer to the suction side of the blades, as noted in the bottom image of Fig. 4. This region extends almost to the middle of the passageway length and it shifts the fluid stream towards the pressure side of the blades.

\section{Radial load on the impeller}

The numerical model was used finally to estimate the radial load on the impeller as a function of flow rate. This was done by storing the unsteady flow computations along one blade passage period. The resulting radial load was calculated by means of a full integration of the instantaneous pressure and shear stress distribution on all the impeller surfaces in each of the time steps.

Fig. 5 presents the average magnitude of the radial load on the impeller when operating in direct (top) and reverse (bottom) mode for all the test flow rates. As observed, the average load in pump mode shows a minimum magnitude at nominal flow rate while increasing for off-design conditions. Its magnitude is especially high at low flow rates and, in particular, at the $20 \%$ flow rate. It is also noted in top of Fig. 5 that the force vector moves towards the fourth quadrant when operating above rated conditions, as expected for a centrifugal pump.

Additionally, Fig. 5 shows an average radial load in turbine mode that increases continuously with flow rate. This average load for $\phi<\phi_{\mathrm{N}}$, however, is not higher than the maximum radial load obtained in pump mode. Thus, it can be concluded that an excessive radial load is not to be expected when the pump operates in reverse mode at moderate flow rates. In contrast, it is seen in Fig. 5 that the load on the impeller increases significantly for reverse operation above rated conditions. Finally, this figure also indicates that the point of application of the force on the impeller is always located at the third quadrant.

The previous figure presented the magnitude of the average radial load on the impeller. However, the results shown in Figs. 3 and 4 suggest that the impeller can be also under the influence of an unsteady (or pulsating) radial load. This unsteady load is caused by the sudden change in pressure experienced by the blades when they pass in front of the volute tongue.
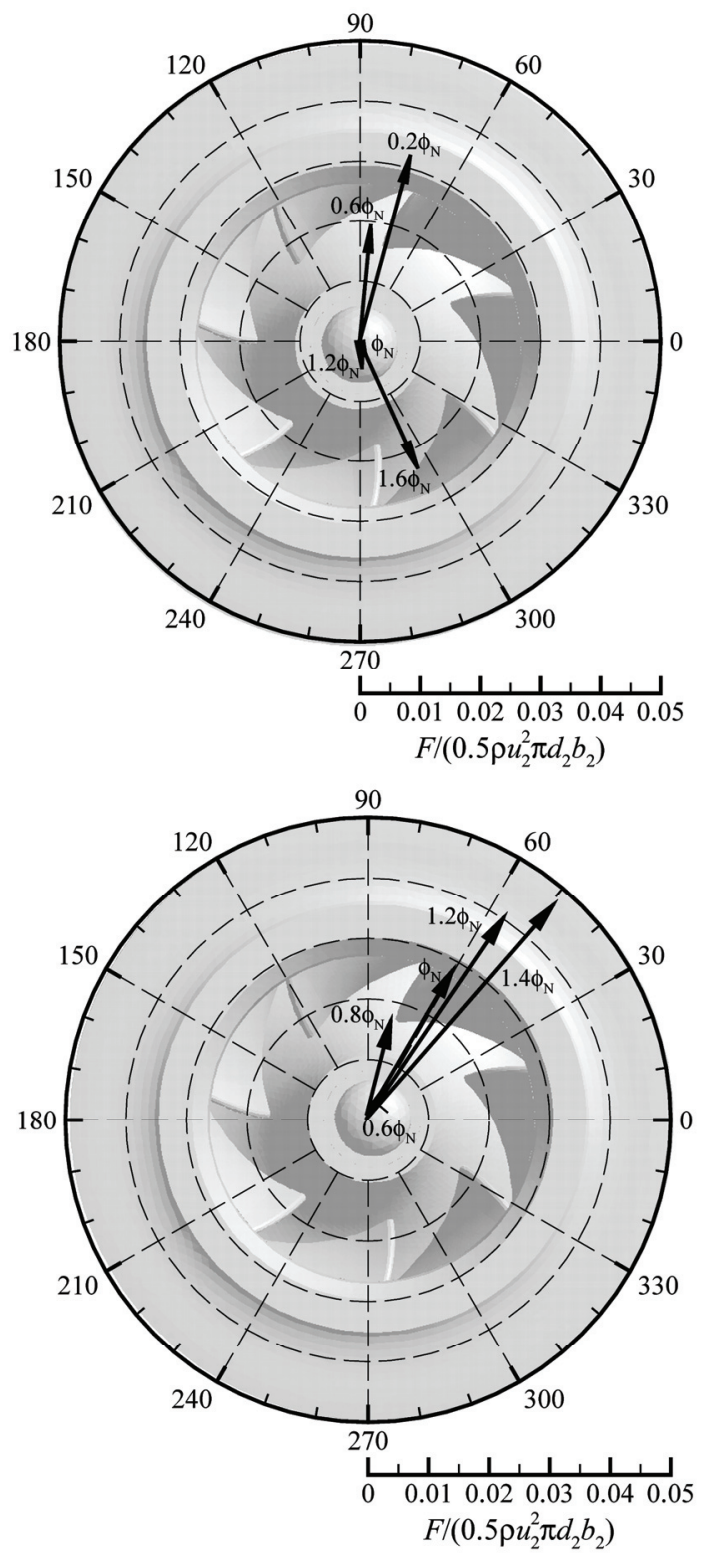

Fig. 5. Average radial load on the impeller as a function of flow rate in direct (top) and reverse (bottom) mode.

The maximum amplitude of the unsteady radial load is presented in Fig. 6 for both modes of operation as a function of flow rate. This amplitude was obtained after subtracting the average value of Fig. 5 from the numerical calculations. As observed, the unsteady load in pump mode follows a similar trend to that previously seen in Fig. 5: it shows a minimum magnitude at nominal flow rate and increasing amplitudes for off-design conditions (especially at the lowest flow rate). It can be also appreciated that the unsteady load can reach a magnitude up to $30 \%$ of the average value.

The trend of the maximum unsteady load in turbine mode also resembles that of Fig. 5, showing increasing amplitudes with increasing flow rates. However, the unsteady amplitude obtained in this mode of operation for moderate flow rates (i.e. for $\phi<\phi_{\mathrm{N}}$ ) results similar or even lower than that estimated in pump mode. In contrast, it is seen in Fig. 6 that the unsteady amplitude in turbine mode is well above the maximum value predicted 
in pump mode for flow rates higher that the rated one. Additionally, it can be appreciated that the maximum unsteady load in reverse mode can reach a magnitude of about $25 \%$ of the average value when operating at medium and high flow rates.

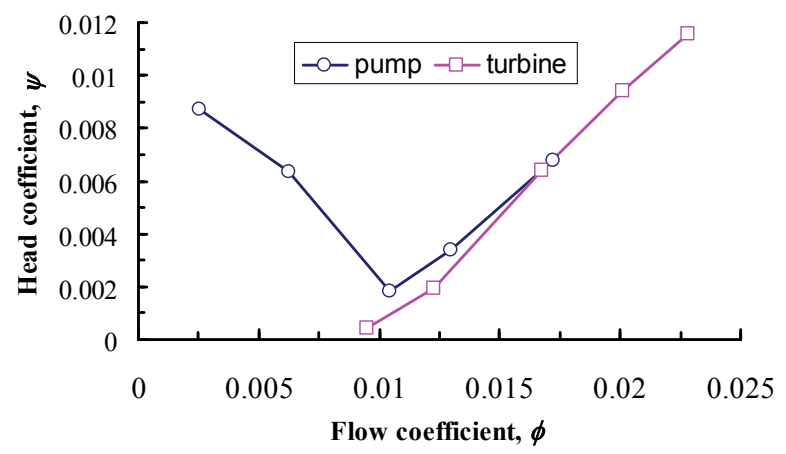

Fig. 6. Maximum amplitude of the unsteady radial load as a function of flow rate.

\section{Conclusions}

The operation of a centrifugal pump working in direct mode and as a centripetal turbine was investigated using a commercial CFD code. The simulations were carried out for five flow rates in each mode of operation after testing the dependence of the numerical predictions with respect to grid and time step size. The predictions were validated with laboratory tests, which showed a good agreement between the experimental and numerical flowhead characteristic.

The numerical model was subsequently used to investigate the global flow in both modes of operation and, additionally, to estimate the radial load on the impeller as a function of flow rate. When operating as a turbine, it was observed that the flow only matched the geometry of the impeller at nominal conditions while developing re-circulating regions of fluid at low and high flow rates. These regions of fluid located either near the pressure side of the blades (flow rates below rated) or near the suction side (high flow rates). The analysis of the flow also revealed the presence of a low pressure region located between the impeller and the tongue.
The predictions of the radial load showed a minimum radial thrust near design conditions in pump mode and an increasing magnitude with flow rate in reverse mode of operation. The magnitude of the total radial load (steady and unsteady components) resulted lower than the maximum total load in pump mode for operating below turbine rated conditions. In contrast, the total thrust on the impeller was found to increase well above this maximum load for flow rates above turbine nominal flow rate. Thus, and considering the usual operation range in centripetal turbines, it can be concluded that the mechanical design of the machine and of the shaft bearings must be carefully undertaken if a usual operation in reverse mode is to be expected.

\section{Acknowledgement}

The authors gratefully acknowledge the financial support of the Ministerio de Educación y Ciencia (Spain) under Project DPI2009-14455.

\section{References}

[1] C.P. Kittredge, "Centrifugal pumps used as hydraulic turbines”, J. Eng. for Power, 1961, series A, pp. 74-77.

[2] B. Neumann, The interaction between geometry and performance of a centrifugal pump, Mechanical Engineering Publications Limited, London (1996).

[3] J. Fernández, E. Blanco, J. Parrondo, M.T. Stickland, and T.J. Scalon, "Performance of a centrifugal pump running in inverse mode", J. Power and Energy, 2004, 218(4), pp. 265271.

[4] K. Majidi, "Numerical Study of Unsteady Flow in a Centrifugal Pump", J. Turbomach., 2005, 127(2), pp. 363-371.

[5] R. Byskov, C.B. Jacobsen, N. Pedersen, "Flow in a Centrifugal Pump Impeller at Design and Off-Design Conditions-Part II: Large Eddy Simulations”, J. Fluids Eng., 2003, 125 (1), pp. 73-83.

[6] M. Zhang and H. Tsukamoto, "Unsteady Hydrodynamic Forces due to Rotor-Stator Interaction on a Diffuser Pump With Identical Number of Vanes on the Impeller and Diffuser", J. Fluids Eng., 2005, 127(4), pp. 743-751.

[7] R. Dong, S. Chu, J. Katz, "Effect of Modification to Tongue and Impeller Geometry on Unsteady Flow, Pressure Fluctuations and Noise in a Centrifugal Pump", J. Turbomach., 1997, 119 (3), pp. 506-515. 